\title{
Transportation Safety Requirements in the Use of Pushchairs on Buses
}

\author{
Juan Dols $^{1}$, Enrique Alcalá ${ }^{2}$ Jaime Masiá $^{1}$, Luis Martínez ${ }^{2}$, Angel Martín ${ }^{2}$ and Antonio Garcia ${ }^{2}$ \\ 1. Institute for Design and Manufacturing (IDF), Universitat Politècnica de València, Spain, Camino de Vera, s/n. 46022 \\ Valencia ,Spain \\ 2. University Institute for Automobile Research (INSIA), Universidad Politécnica de Madrid, Spain, Campus sur de la UPM, Ctra. de \\ Valencia, km 7. 28031 Madrid, Spain
}

\begin{abstract}
The safe use of buses by passengers travelling with pushchairs for young children is a mobility problem that has not yet been satisfactorily resolved. The lack of national and international standards in this area in the past led to the development of a research project developed jointly by the Universitat Politècnica València and the Universidad Politècnica Madrid (Spain). This paper shows a comparative study to evaluate the dynamic behaviour in case of low-g accidents reproducing frontal impacts resembling real traffic events (deceleration $2 \mathrm{~g}, \Delta \mathrm{V} 20 \mathrm{~km} / \mathrm{h}$ ) of a new pushchair restraint system against the use of back-restraint by pushchairs as establishes R 107- 06 series of amendments. In the dynamic trials, three pushchair restraint prototypes and a typical wheelchair back-restraint system combined with two worst-case up-to-date pushchair models in misuse and correct use configurations were tested. The results demonstrated the need for preventing children injuries as a consequence of low-g accidents. The new design meets and improves the latest revision of regulation R107 regarding the use of restraint systems for the transport of wheelchair and pushchair passengers and represents an advance for pushchairs travelling on buses.
\end{abstract}

Key words: Transportation safety, pushchairs, children injury, restraint system, public transport.

\section{Introduction}

The use of public transport is a need for all citizens. However, there are still some groups who experience difficulties moving on buses, such as users who travel with pushchairs or strollers. These users also face safety troubles as it is often necessary for children to be taken out of their pushchairs and held in the arms of an accompanying person. From the standpoint of operating companies, the use of buses by this group represents a design problem that has not yet been satisfactorily and effectively resolved.

Little research has been published on the use of buses by passengers travelling with pushchairs. Unfortunately, this type of transport has not been fully analysed and neither research has been developed in the field of

Corresponding author: Juan Dols, associate professor; research fields: transport system, traffic management, transportation safety, and vehicles design. E-mail: jdols@mcm.upv.es. accessible transport nor statistical studies have been conducted by national administrations to collect incidents and accidents during movement of passengers with pushchairs in public transportation vehicles.

Ref. [1] shows one of the few studies published for determining the safety requirements in the use of pushchairs on buses. This study was the result of the ASUCAR (determination of accessibility and safety requirements in the use of children's pushchairs in public transportation vehicles) research project: "Determination of Accessibility and Safety Requirements in the use of Pushchairs on Buses", sponsored by the Spanish Ministry of Science and Innovation under the National Science and Research Plan 2008-2011, and developed jointly by the IDF (Institute for Design and Manufacturing) from UPV (Universitat Politècnica de València) and the INSIA (Institute for Automobile Research) from UPM (Universidad Politècnica de Madrid) (Spain). In 
relation with the same project, Ref. [2] shows the results obtained in practical field and naturalistic driving tests in which were measured the dynamic behaviour of pushchairs following various types of common bus manoeuvres, and lead to the conclusion that in some trip situations and configurations, there is a need for preventing children injuries travelling in unfolded pushchairs as a consequence of low-g impacts when the occupied pushchair hits interior parts of the vehicle - as the aisle, floor, doors or other dangerous obstacles-when launched after the deceleration movement. In that research, it was seen anemergencybraking as the bus manoeuver worst-case in which the highest values of longitudinal acceleration for a pushchair was found: around $1 \mathrm{~g}\left(1 \mathrm{~g}=9.81 \mathrm{~m} / \mathrm{s}^{2}\right)$, for a restrained pushchair and around $6 \mathrm{~g}$ for pushchairs unrestrained, where it strucks violently against objects in the inner bus. Data obtained in these field tests allowed also to determine the minimum loads that must be withstood to restrain a pushchair during normal vehicle driving conditions. Considering a maximum pushchair mass of $20 \mathrm{~kg}$, a maximum child mass of 20 $\mathrm{kg}$, and a maximum longitudinal deceleration of $1 \mathrm{~g}$ during braking and $2 \mathrm{~g}$ in minor collisions, the minimum resistive load was estimated at about $800 \mathrm{~N}$ during braking and 1,600 $\mathrm{N}$ in minor collisions.

As a result, this unresolved safety issue motivated the development of a new concept of restrain system for pushchairs when travelling unfolded with a child on board, and made the relevant application for a patent No. P-201131557 [3]. By the time in which the project was developed, it was observed the absence of any specific restraint system for a pushchair when travelling on a bus simultaneously with a wheelchair user. Indeed, the only type of restraint system regulated in European legislation [4] and UNECE regulations [5] is designed for wheelchairs and takes the form of back restraint walls.

\subsection{The New Pushchair Restraint System Design}

According to ASUCAR project, it was developed a
Code of Good Practice [1] to determine the most suitable locations inside a bus for travelling with unfolded pushchairs, specifying three possible areas on interior updated buses design as follows (Fig. 1):

- Area 1: area provided for wheelchair passengers, in which pushchairs and wheelchairs can use the same space and restraints, provided they do not ride in the vehicle at the same time. In both cases, the adult in the wheelchair and the child in the pushchair are supported by rear wall and face backwards (Fig. 1a). In this position, seatbelts are unnecessary for the pushchair (however, it is recommended that brakes are applied). This area is considered the best choice;

- Area 2: area booked for pushchairs near the central door of the bus in which the pushchair is located next to a cross bar separating the central interior space from the first row of rear seats (Fig. 1a). The pushchair faces forwards. In this position, a seat belt restraint system and the application of the pushchair brake is recommended. This area is recommended for using when a wheelchair passenger is already occupying the area indicated for wheelchair users, or when two or more pushchairs are travelling at the same trip;

- Area 3: area between Zones 1 and 2 (Figs. 1a and 1b). In this case, it is recommended that the pushchair travel facing backwards with brakes applied to prevent sliding or tipping sideways. This is the area recommended for the installation of the new pushchair restraint developed in the ASUCAR project.

So, a new concept of restraint system consisting of a folding back-rest panel fixed to the sidewall of the bus was developed [3]. The back-rest is composed by a double panel that folds to the side of the bus when not in use, and when needed, unfolds $90^{\circ}$ around a vertical axis to become locked perpendicularly to the wall to support a pushchair (Fig. 2). Once the main panel is deployed, a second panel also unfolds by $90^{\circ}$ to provide side support for the pushchair and prevent sideways movement during bus manoeuvres. With this device, that pushchair must travel facing rearwards. 


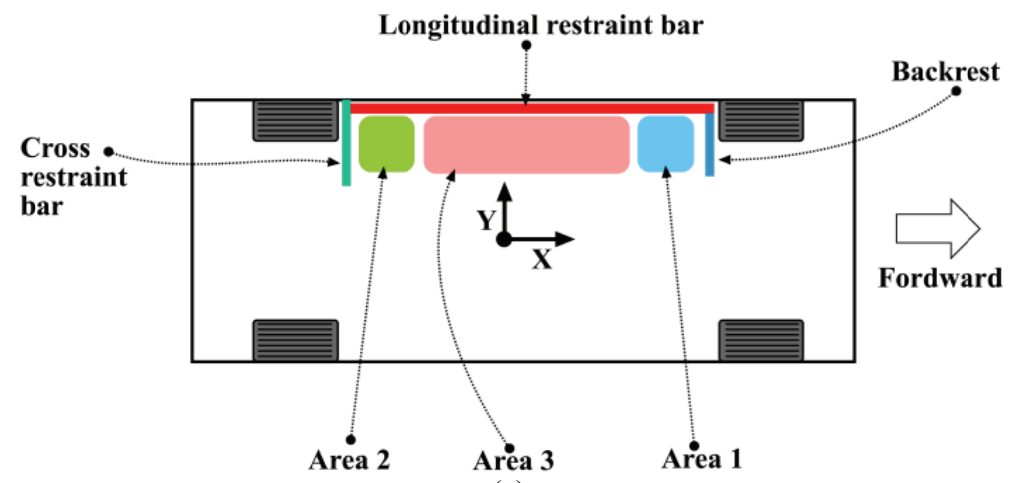

(a)

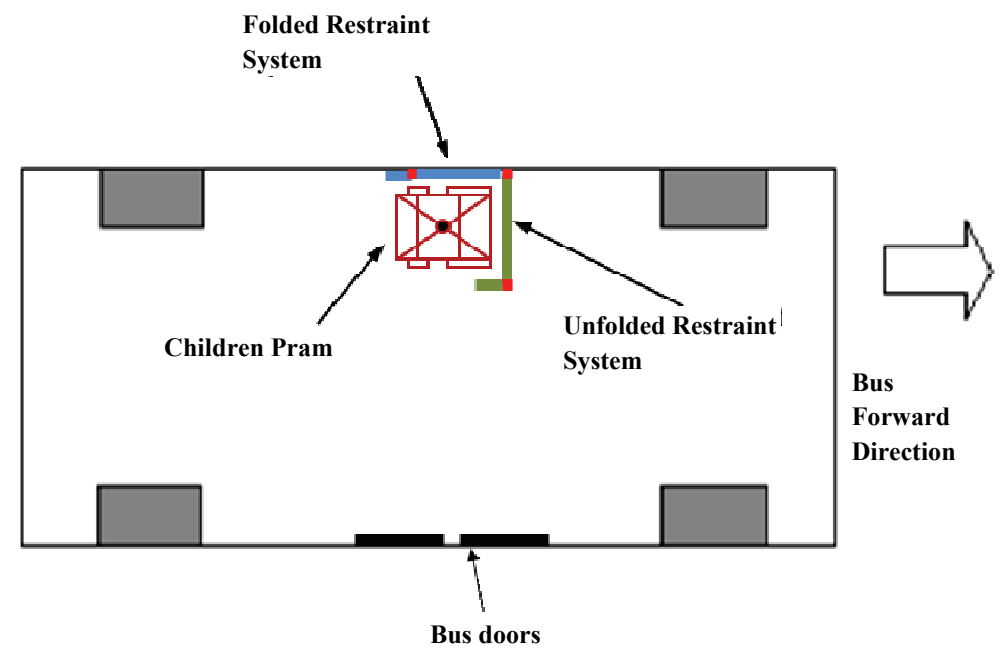

(b)

Fig. 1 Interior Locations for travelling with unfolded pushchairs in public transportation buses: (a) areas of available location of the pushchair inside the bus; (b) location of pushchair restraint system in Area 3.

\subsection{Legal Framework}

Recently, the Working Party on General Safety Provisions (GRSG) of the World Forum for Harmonization of Vehicle Regulations (WP29 of the UN) approved a proposal to amend Regulation 107 [6] and adapt a specific area on buses (Class I) for children to sit in their pushchairs during transport. This proposal enables that space for wheelchairs users (in rigid buses) is shared with children in pushchairs by using backrests and wall elements (armrests or panel bars) to retain pushchairs. In case of articulated buses, or specially designed vehicles, the proposal establishes a space for a pushchair that measures at least $750 \mathrm{~mm}$ in width and $1,300 \mathrm{~mm}$ in length - and separated from the space used by wheelchair passengers.
Regarding the requirements to ensure the stability of pushchairs, the proposal provides for the revision of Annex 8 of Regulation 107 to enable one of the sides of the space for pushchairs to be conditioned so that pushchairs could be positioned at the side of the vehicle with the front of the pushchair (or baby carriage) in contact with a support or backrest that is perpendicular to the longitudinal axis of the bus (and which could coincide with the panel installed for wheelchair passengers). Retractable armrests or other equivalent devices could be used on the side opposite the pushchair to prevent or restrict sideways movement.

The publication of this proposal [6] raises questions as to whether pushchairs can withstand the same forces as wheelchairs, or if the stiffness and strength requirements for restraints are the same for both groups 
or what would happen if a space for a pushchair is already occupied by a wheelchair. Similarly, the revision to Regulation 107 does not determine whether pushchairs should be facing forwards or backwards, or whether they should be using the back-restraint as for wheelchairs or not.

This paper shows the results of a comparative study to evaluate the new ASUCAR pushchair restraint system against the use of backrest by pushchairs as establishes R 107-06 series of amendments in case of low-g accidents. An analysis of the results obtained in the dynamic low-g testing has confirmed the effectiveness of the restraint system in circumstances similar to real traffic conditions and establishes a measurement of the degree of compatibility between the pushchair restraint system and that used by wheelchair passengers based on back-restraints, giving advice on how to proceed with their use and highlighting the degree of policy and legislative coherence with the latest proposals for R 107 .

\section{Methodology}

The comparative study between the ASUCAR pushchair restraint system and the backrest wheelchair system - used by a pushchair-was based on the development of a series of crash tests based on the dynamic requirements obtained in previous phases of the project [2]. As there is no regulation that enables the homologation of the new restraint system, all the tests were made for research purposes - and therefore the sled deceleration was based on these criteria. Various restraint system prototypes were manufactured to perform the tests, and their strength and dynamic behaviour was compared with the use of back-restraint panels for wheelchair passengers.

\subsection{Test Samples}

Different samples were used for performing the impact tests on the sled. The rear wall restraint panel for wheelchair passenger was representative of those used in city buses, and was provided by the public bus authority in Madrid (Fig. 3). In the test, the panel was rigidly anchored to a wall at one end of the sled. In normal practice, these panels are fastened to vertical bars that, in turn, are anchored to the floor and ceiling of the vehicle body. Prototypes of the ASUCAR restraint system consisted of folding panels with metal frame and covered with wooden planks (Prototype No. 1 with height $500 \mathrm{~mm}$, and Prototype No. 2 with height $400 \mathrm{~mm}$ ), or metal frame covered with wooden planks and padded with exterior trim (Prototype No. 3 with height $400 \mathrm{~mm})$.

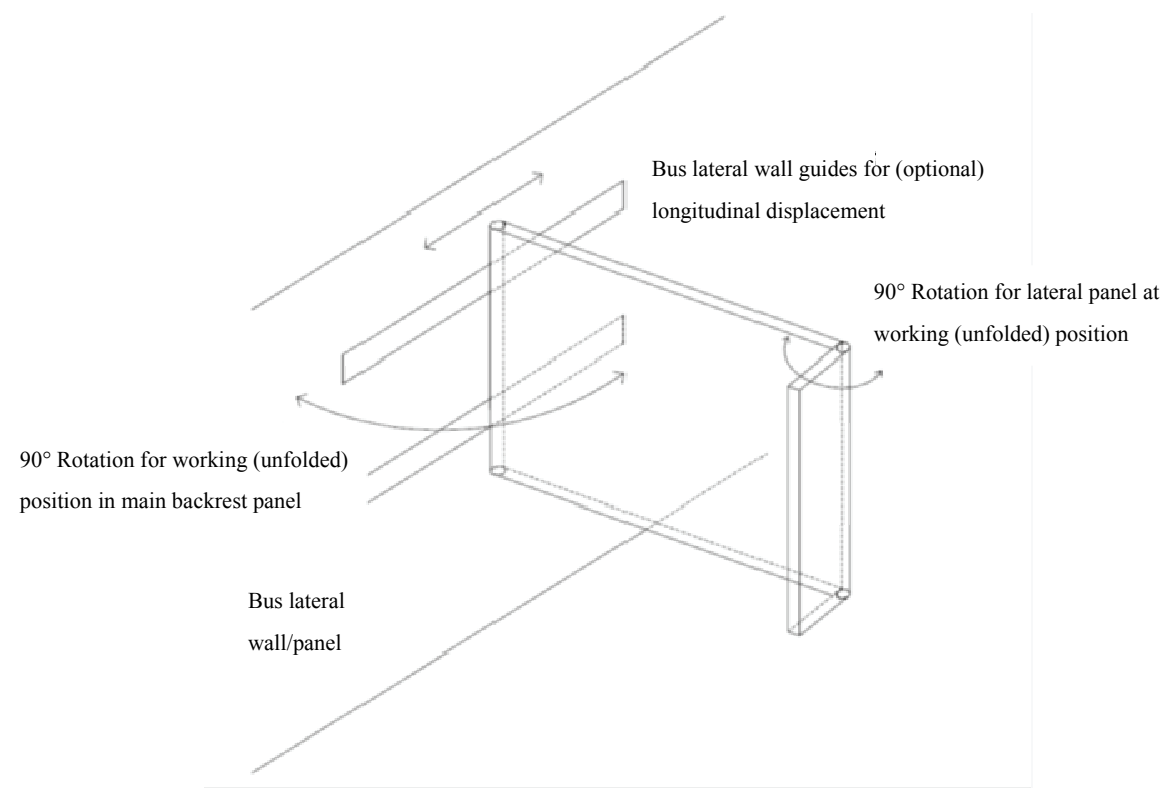

Fig. 2 Configuration for transporting an unfolded pushchair using the ASUCAR restraint system. 


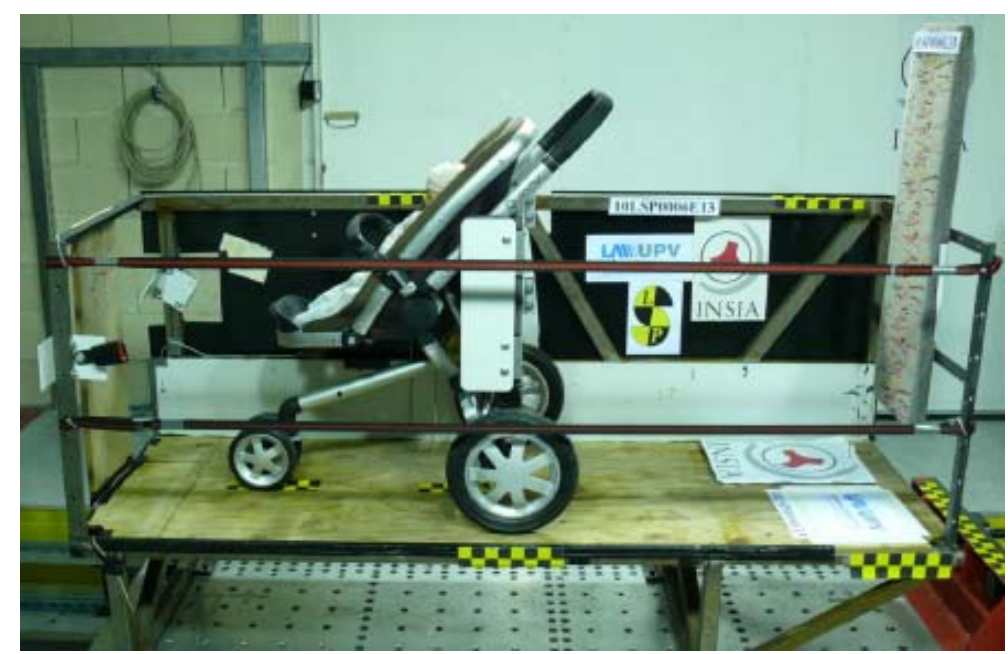

Fig. 3 Rear wall restraint panel for wheelchair passenger and pushchair restraint system mounted on the sled.

The pushchair samples were selected based on the field results obtained in previous phases of the ASUCAR research project representing the worst-case market models. In this paper are nominated as (Fig. 3):

- Model SX. Pushchair with adjustable height. It has four independent wheels, with front-wheel steering and brakes on the rear wheels;

- Model QB. Seat angle adjustable and used with the most upright position. It has four wheels, with twin front wheels and brakes on rear wheels.

A tri-axle accelerometer was fitted to each of the tested pushchairs to record the deceleration. The position of the tri-axle accelerometer and orientation of the coordinated acceleration measurements for each pushchair can be seen in Fig. 4.

\subsection{Test Description}

Dynamic tests were performed on a deceleration sled that was accelerated to the speed designated in the test conditions $(\approx 20 \mathrm{~km} / \mathrm{h})$ and programmed to stop $(\approx 2 \mathrm{~g})$ in a controlled manner. The level of severity for the low-g deceleration was equivalent to a sudden hard braking manoeuvre suffered by a city bus as obtained in Ref. [2].

The test facility belonged to the INSIAat the Universidad Politécnica de Madrid (Spain), and the testing took place between July and September, 2013. The pushchair restraint was mounted on a module representing the floor and side of a city bus adapted for wheelchair passengers (Fig. 3). All the tests were performed with the pushchair facing backwards. Longitudinal deceleration of the sled was measured by an accelerometer according to SAE J211 [7] including initial speed and the movement near the brake point. Fig. 5 shows an example of the deceleration pulse applied in the sled.

The kinematics of the trial was recorded with two high speeds video cameras (1,000 frames per second), and a third conventional camera operating at $30 \mathrm{fps}$.

Two types of crash dummies were used in the tests. In a first-trial test, a TNO P3 dummy representing a three-year-old child was used and equipped with two tri-axle accelerometers: one installed in the head (filter CFC_1000) and another in the chest (filter CFC_180).

In other tests, a Q1 dummy was used to simulate an one-year-old child. The dummy was equipped with sensors to measure tri-axle acceleration in the head (CFC_1000), chest (CFC_180), pelvis (CFC_1000); and a load cell in the upper neck measured forces (CFC_1000) and moments (CFC_600), as well as chest deformation (CFC_600). These dummies were approved for the implementation of $\mathrm{UN}$ regulations 129R00 and 44R04 [8] for the homologation of child restraints. At the time of the study was made there was no wide-ranging international standard injury criteria for the Q1 dummy. Therefore, the injury criteria used 


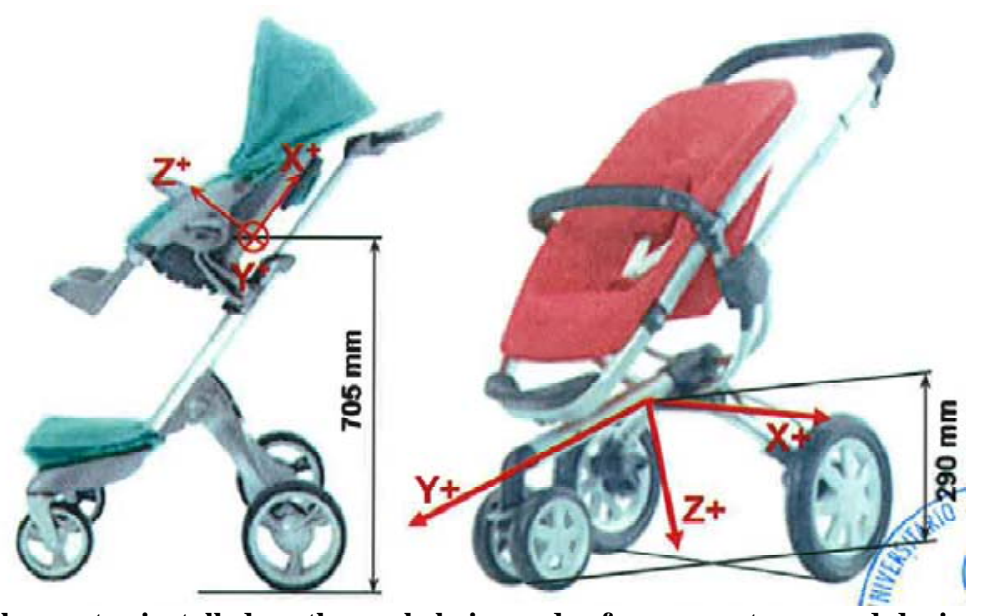

Fig. 4 Position of the accelerometer installed on the pushchairs and reference systems used during dynamic tests (left: SX; right: QB).

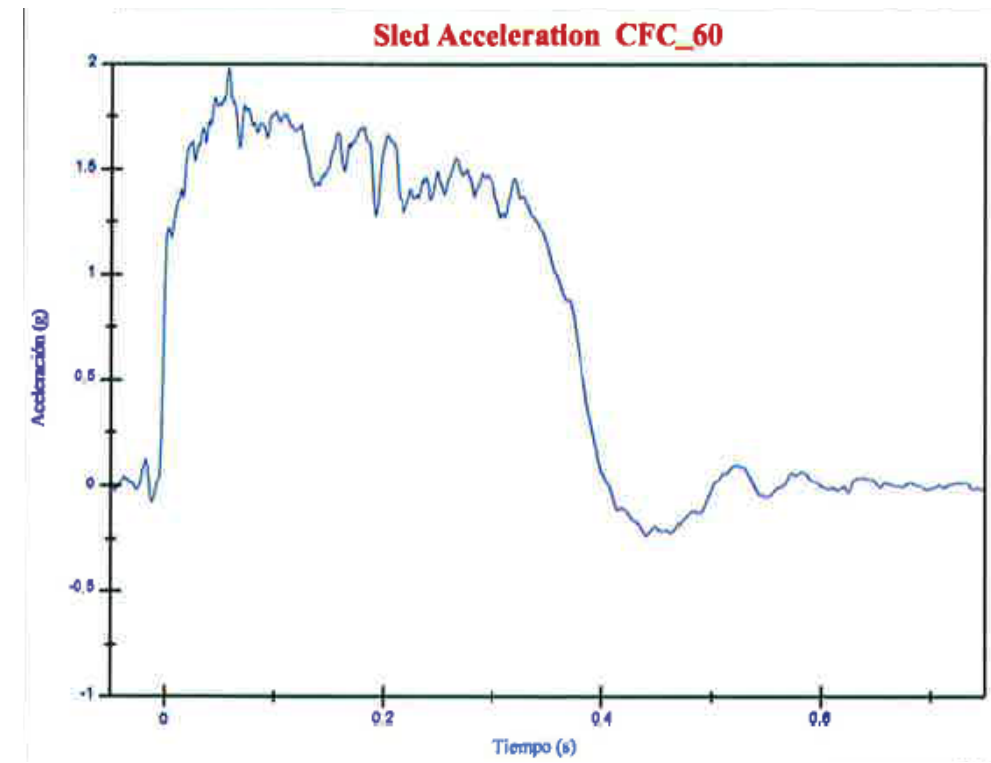

Fig. 5 Deceleration pulse applied to the sled platform.

for the Q1 dummy were obtained by scaling the information in Regulation 94R01 [9], and applying the parameters included in the work of Ref. [10]. The testing configuration and main features of the tests are described in Table 1.

The purpose of dynamic test planning was to detect "misuse" and "correct use" configurations, as well as to deal with the behaviour between the use of pushchairs with the ASUCAR restraint system and backrest restraint system-compatible with wheelchair travellers-based on R 107 amendments [6]. All tests were representing worst-case configurations in relation with pushchair model, pushchair orientation, impact direction, pushchair brakes applied and distance from pushchair to restraint system. As a result, it was defined the following type of tests:

- Test E01: The objective of this test was to verify the structural strength of the pushchair panel prototype Num. 1 using the heaviest dummy (TNO type P3-15 kg weight). This configuration was considered as a "misuse" as it aimed to verify the behaviour of the least stable pushchair, with the tallest panel prototype, and without brakes applied to the wheels. The pushchair was placed facing backwards in its lowest position. The free flight distance between the rear wheels and the restraint system (panel prototype Num. 1) was $89 \mathrm{~mm}$. 
Table 1 Testing configuration for dynamic impact tests.

\begin{tabular}{llllllll}
\hline Test identification & $\begin{array}{l}\text { Impact } \\
\text { direction }\end{array}$ & $\begin{array}{l}\text { Pushchair } \\
\text { orientation }\end{array}$ & $\begin{array}{l}\text { Restraint system } \\
\text { prototype }\end{array}$ & Dummy & $\begin{array}{l}\text { Pushchair } \\
\text { model }\end{array}$ & $\begin{array}{l}\text { Pushchair } \\
\text { brakes applied }\end{array}$ & $\begin{array}{l}\text { Distance from pushchair } \\
\text { to restraint system (mm) }\end{array}$ \\
\hline E01 urban bus module & Frontal & FB & Prototype \#1 & TNO P3 & SX & No & $89 \mathrm{~mm}$ \\
E02WBRS & Frontal & FB & WBRS & Q1 & SX & No & $150 \mathrm{~mm}$ \\
E03 & Frontal & FB & Prototype \#2 & Q1 & SX & No & $150 \mathrm{~mm}$ \\
E04 & Frontal & FB & Prototype \#3 & Q1 & QB & Yes & $0 \mathrm{~mm}$ \\
E05 & Frontal & FB & Prototype \#3 & Q1 & QB & No & $150 \mathrm{~mm}$ \\
E06WBRS & Frontal & FB & WBRS & Q1 & QB & Yes & $0 \mathrm{~mm}$ \\
\hline
\end{tabular}

WBRS — wheelchair backrest system, FB — facing backwards, TNO P3 — child dummy TNO type P3 (3 years old), Q1 —child dummy type Q1 (1 year old).

The panel height was $500 \mathrm{~mm}$;

- Test E02: The aim of this test was to verify the structural strength and behaviour of a back-restraint for wheelchair passengers using the instrumented Q1 dummy (representing an one-year-old child). This configuration is considered as "misuse" as it was intended to verify the behaviour of the least stable pushchair when interacting with the panel used by wheelchair passengers. In this case, we used the SX pushchair facing backwards in its highest position, with a distance from the pushchair grab-bar to the panel (in a horizontal position) of $150 \mathrm{~mm}$ - and without brakes applied to the wheels;

- Test E03: The aim of this test was to verify the structural strength of the prototype panel Num. 2 using the Q1 instrumented dummy. These conditions represented a "misuse" because the SX was the least stable pushchair and was positioned facing backwards in its highest position with a distance of the centre bar to the panel of $150 \mathrm{~mm}$ without brakes applied to the wheels;

- Test E04: This test aimed to verify the structural strength of Num. 3 prototype panel for pushchairs using the Q1 dummy. This configuration was considered as "misuse". The QB pushchair faced backwards with the side bar of the pushchair touching the panel - and with brakes applied to the wheels;

- Test E05: This test was intended to verify the structural strength of Num. 3 prototype wheelchair rear wall restraint system using the Q1 dummy. This configuration is considered a "misuse" as it examined the behaviour of the backward facing QB pushchair when interacting with the Num. 3 panel when the sidebars of pushchair were $150 \mathrm{~mm}$ from the panel - and without brakes applied to the wheels;

- Test E06: Finally, this test aimed to verify the structural strength and behaviour of the panel for wheelchair passengers using the Q1 dummy. This configuration is considered "correct use" as it verified the behaviour of the widest pushchair when interacting with the wheelchair back-restraint system. In this case, the QB chair faced backwards with the handle (horizontal) touching the wheelchair back-restraint system, and brakes applied to the wheels.

It should be noted that the goal of Tests E02 and E06 was not only to verify the structural strength of the new pushchair restraint, but also to compare dynamic behaviour in settings of correct use and misuse in relation to the technical requirements defined in the modification to Regulation 107 [6]. So, the test configuration scheduled gave us the opportunity to develop a qualitative comparative study between different configurations as:

- Analysis of E02 in comparison with E03. In this case, two test configurations classified as misuse were compared. The least stable pushchair (the SX) behaviour was compared to the wheelchair back-restraint system and the ASUCAR prototype No. 2;

- Analysis of E04 compared with E05. In this case, two configurations were tested using one of the pushchair widest models with twin front wheels (QB). 
The analysis compared a "correct use" configuration (E04) with pushchair in contact with the ASUCAR prototype No. 3 and wheel brakes applied, with a "misuse" application (E05) where the wheelchair faced backwards, with a distance of $150 \mathrm{~mm}$ to the restraint panel, and wheel brakes not applied.

\section{Results}

The battery of dynamic tests produced injury criteria and various parameters which were recorded in the dummy sensors. Injury criteria are fundamentally developed for adult-sized manikins (specifically the Hybrid III 50th male). The reference values on infantile manikins must be scaled from the data of the average adult occupant.

Although the same instrumentation as that required by R44 [8] has been used on the P3 manikin, none of the criteria for injury to this manikin on R44 have been used since the direction of impact is opposite to that found in the tests of this regulation. Therefore, the injury criteria of R44 are not fully applicable to the test configurations performed in this test. Table 2 shows the results obtained in the E01 test, in which the TNO P3 dummy was used, and in which time elapsed as well as maximum and minimum values accelerations were obtained. To facilitate an understanding and interpretation of the results in the other trials in which the instrumented Q1 dummy was used, the values obtained in each test are presented in Table 3 as percentages of the defined maximum values of the injury criteria as established in the research.
Criteria regarding child injury were used to test the "goodness" of the data obtained. To achieve this, scaling factors from Ref. [10] were used as these currently enjoy the widest recognition worldwide. These scaling factors have been applied in the reference values used in the United Nations regulation 94R01 [9] for average sized adults and for the FMVSS (Federal Motor Vehicle Safety Standards) regulations. The "limit values" established in these regulations corresponding to a certain probability of injury for various body parts.

Traditionally, United Nations (or European) regulations set limits slightly more stringent than US regulations. Proof of this is that the values defined in Ref. [10] that match parameters measured in regulation 94R02 have wider values. So, the research team considered it worthwhile using the parameters derived from European regulations for two main reasons: these parameters are on the safe side (the lesion limit values obtained are lower than those used by FMVSS values), and there is congruence between the regulations applied and the region where the buses are being used. To aid the diffusion and validity of the study, the criteria arising from injury limits included in American legislation are calculated - as these include expanded injury criteria to the neck area.

\section{Discussion}

An analysis of the results obtained in the dynamic tests has drawn a series of discussion ideas for both misuse and correct use configurations:

Table 2 Maximum and minimum value obtained in test E01 with TNO P3 dummy.

\begin{tabular}{lllllll}
\hline \multirow{2}{*}{ Description } & \multirow{2}{*}{ Units } & \multicolumn{2}{c}{ Maximum measured } & \multicolumn{2}{c}{ Minimum measured } \\
\cline { 4 - 7 } & & & Value & $\mathrm{T}(\mathrm{ms})$ & Value & $\mathrm{T}(\mathrm{ms})$ \\
\hline \multirow{3}{*}{ Head } & Acceleration X & & 16.61 & 251.55 & -0.2 & 42.35 \\
& Acceleration Y & $\mathrm{g}$ & 1.92 & 569.05 & -0.3 & 971.85 \\
& Acceleration Z & & 13.92 & 261.2 & -1.23 & 141.4 \\
& Resulting acceleration & & 21.2 & 251.2 & - & - \\
\multirow{3}{*}{ Chest } & Acceleration X & & 5.8 & 251.2 & -0.49 & 908.85 \\
& Acceleration Y & $\mathrm{g}$ & 2.51 & 534.25 & -1.17 & 150.45 \\
& Acceleration Z & & 5.17 & 317.55 & -1.63 & 141.8 \\
& Resulting acceleration & & 6.21 & 249.15 & - & - \\
\hline
\end{tabular}


Table 3 Results obtained in comparison with the injury criteria in the rest of tests with Q1 dummy.

\begin{tabular}{|c|c|c|c|c|c|c|c|c|}
\hline \multirow{2}{*}{ Dummy part } & \multirow{2}{*}{ Parameter } & \multirow{2}{*}{ Unit } & \multirow{2}{*}{ Maximum value $e^{(* *)}$} & \multicolumn{5}{|c|}{ (\%) with respect to the maximum value registered in each test } \\
\hline & & & & E02 & E03 & E04 & E05 & E06 \\
\hline \multirow{4}{*}{ Head } & $\mathrm{HIC}_{15}$ & & 389.00 & 3.50 & 1.20 & 0.33 & 6.11 & 0.25 \\
\hline & $\mathrm{HPC}\left(\mathrm{HIC}_{36}\right)^{*}$ & & 555.71 & 2.46 & 0.84 & 0.29 & 4.28 & 0.34 \\
\hline & Acceleration of CG & $\mathrm{g}$ & 154.00 & 12.60 & 11.78 & 4.21 & 21.64 & 3.55 \\
\hline & Acceleration $3 \mathrm{~ms}^{*}$ & $\mathrm{~g}$ & 68.44 & 27.55 & 21.21 & 9.29 & 40.78 & 7.91 \\
\hline \multirow{13}{*}{$\begin{array}{l}\text { Neck } \\
\text { (upper neck) }\end{array}$} & Shear force $F x^{*}$ & $\mathrm{~N}$ & \pm 740.00 & 16.39 & 10.04 & 9.71 & 22.77 & 3.15 \\
\hline & Tension force $+F z^{*}$ & $\mathrm{~N}$ & 783.45 & 11.51 & 10.14 & 2.39 & 17.18 & 3.88 \\
\hline & Compressive strength $-\mathrm{Fz}$ & $\mathrm{N}$ & -960.00 & 6.15 & 4.48 & 1.01 & 8.04 & 1.40 \\
\hline & Lateral moment $M x$ & $\mathrm{Nm}$ & \pm 21.00 & 8.00 & 3.19 & 4.38 & 3.95 & 1.38 \\
\hline & Bending moment $+M y$ & $\mathrm{Nm}$ & 27.00 & 7.00 & 2.26 & 3.22 & 10.96 & 1.07 \\
\hline & Extension moment $-M y^{*}$ & $\mathrm{Nm}$ & -8.31 & 112.72 & 94.80 & 58.47 & 126.44 & 44.15 \\
\hline & Yaw moment $M z$ & $\mathrm{Nm}$ & \pm 14.00 & 13.71 & 4.93 & 9.36 & 10.64 & 3.57 \\
\hline & $\mathrm{N}_{\mathrm{ij}}$ (traction bending) & & 1.00 & 5.44 & 2.85 & 2.41 & 8.59 & 0.81 \\
\hline & $\mathrm{N}_{\mathrm{ij}}$ (traction-extension) & & 1.00 & 41.10 & 44.57 & 20.81 & 59.94 & 21.54 \\
\hline & $\mathrm{N}_{\mathrm{ij}}$ (compression-bending) & & 1.00 & 5.27 & 2.14 & 0.69 & 5.36 & 1.36 \\
\hline & $\mathrm{N}_{\mathrm{ij}}$ (compression-extension) & & 1.00 & 54.34 & 39.28 & 26.40 & 56.30 & 10.68 \\
\hline & NIC traction & & Corridor & 11.39 & 10.83 & 5.18 & 16.99 & 8.20 \\
\hline & NIC shear & & Corridor & 18.41 & 10.32 & 14.37 & 23.81 & 7.07 \\
\hline \multirow{2}{*}{ Chest } & Sternum movement, $D x^{*}$ & $\mathrm{~mm}$ & 24.00 & -3.38 & -4.38 & 0.46 & 3.08 & 0.08 \\
\hline & Acceleration T4 (resulting) & g & 87.00 & 13.70 & 16.54 & 5.25 & 21.51 & 3.98 \\
\hline
\end{tabular}

*: peak values obtained from ECE R94 and scaled for Q1 dummy. Note: All data refers to peak values, except for 3 ms acceleration; $* *$ : maximum values established from scaling factors as described in Refs. $[9,10]$.

- Test E01: This configuration was considered as "misuse" and aimed to verify the behaviour of the least stable pushchair (SX) when interacting with the tallest prototype No. 1 without brakes applied to the wheels. After initiating deceleration, the rear wheels of the pushchair were the first point to contact with the panel $(150 \mathrm{~ms})$, and this caused a backward rotation of the pushchair as the centre of gravity was above the contact point. This initial backward rotation of the pushchair produced a second impact of the central mast of the pushchair $(275 \mathrm{~ms})$ which generated a rotational movement around the mast that tended to push the pushchair into the aisle. This restraint configuration produced unsatisfactory results with respect to safety. Following the results obtained in this test, it was decided to modify the design of the prototype panel by reducing its height to $400 \mathrm{~mm}$. This solution was also adopted for panel prototypes numbers 2 and 3. The structural strength of the No. 1 prototype panel was satisfactory due to only a small permanent deformation appeared at the top of the joint with the wall of the module (vehicle). After testing, the panel continued working properly and no tears or sharp edges were observed. The maximum values obtained for the dummy's head and chest were far from the maximum limit values defined by the criteria for child injuries in R44 [8];

- Test E02: This configuration was considered as "misuse" as the aim is to verify behaviour of the most unstable pushchair when interacting with the panel for wheelchair passengers. During impact, the pushchair - with a $150 \mathrm{~mm}$, gap between the pushchair and the panel - made contact $250 \mathrm{~ms}$, after deceleration began. The first contact was produced by the horizontal handgrip of the pushchair, which was unable to withstand the force of impact and bent downwards. This caused the pushchair to continue its backward movement until a second contact occurred, namely, the rear wheels struck the wheelchair back-restraint (400 $\mathrm{ms})$. Nevertheless, the stability of the back-restraint was enough and the pushchair remained upright. Fig. 6 shows pushchair kinematics during the impact phase. 
During the rebound of the pushchair after striking the wheelchair back-restraint, the pushchair flew upwards and towards the central aisle of the bus. The rotational inertia occurred because the pushchair (SX) has a telescopic central axis on which the chair rested, rotating around this axis when it stuck the rear wall.

- The maximum values produced in the dummy's head and chest, were far from the limits defined by the child injury criteria. However, the neck was extended in the $\mathrm{Y}$ direction such that values exceeded the allowed values by $12.7 \%$ (9.37 $\mathrm{Nm}$ compared to a tolerance limit of $8.31 \mathrm{Nm}$ ). This peak extension was produced just as the handle of the pushchair hit the wheelchair rear wall, and caused the highest values of $N_{i j}$ (traction-extension) and $N_{i j}$ (compression-extension), with values of $41.10 \%$ and $54.34 \%$ of the possible limit values. Nevertheless, it was considered that the behaviour of the pushchair and the wheelchair back-restraint system was correct in retaining the pushchair during impact;

- Test E03: This test was considered "misuse" and the aim was to verify the behaviour of the least stable pushchair when interacting with the No. 2 prototype. During the test, there was a free movement of the pushchair until striking the panel $(155 \mathrm{~ms})$. As the wheels of the pushchair passed under the folding panel, the first contact occurred with the central telescopic rod on which the chair was mounted. During the rebound phase after striking the panel, the pushchair remained in a horizontal position and moved backwards without spinning on its vertical axis, and without moving towards the central aisle of the bus.

The maximum values produced in the dummy's head and chest were far from the limits defined by the criteria for child injury. However, an extension movement was produced in the neck in the $\mathrm{Y}$ direction, which almost reached the limit value $(7.88 \mathrm{Nm}$ compared to a limit of $8.31 \mathrm{Nm}$ ). This peak was produced when the telescopic bar of the pushchair struck the panel, and produced the highest values of $N_{i j}$ (traction-extension) and $N_{i j}$ (compression-extension), with values of $44.57 \%$ and $39.28 \%$ (well within value limits). It was considered that the behaviour of the pushchair with the panel prototype No. 2 was satisfactory and the pushchair was retained properly during impact;

Test E04: This configuration represented a "correct use". The results showed that the restraint was adequate, since the pushchair remained upright at all times. Since there was no relative velocity between the pushchair and the prototype No. 3 the stability was retained, although there was a small yaw effect during the rebound. As the pushchair wheels had the brakes applied, the forward movement due to the elasticity of the panel was reduced, and the panel absorbed part of the movement, neither the pushchair revolving around its vertical axis nor moving towards the central aisle.

The maximum values produced on the dummy for both the head and chest were far from the limits defined by the child injury criteria. The only significant value occurred in the neck extension movement in the $\mathrm{Y}$ direction, which almost reached a value of $4.86 \mathrm{Nm}$ compared with a tolerance limit of $8.31 \mathrm{Nm}$ (or $58.47 \%$ ), and therefore far from the tolerance limit. It was considered that the behaviour of the pushchair with the No. 3 prototype was very good in ensuring retention of the pushchair during impact;

- Test E05: This configuration was considered as "misuse" and our aim was to verify the behaviour of the QB pushchair when interacting with the prototype No. 3. The separation between the side bar of the pushchair and the panel caused a free movement of the pushchair during $170 \mathrm{~ms}$ until striking the panel. First contact occurred on the right-side bar of the pushchair and a second contact was made immediately afterwards with the left bar since the wheels of the pushchair passed under the folding panel.

The stability offered by the restraint system was satisfactory, since the pushchair maintained its upright position. During the rebound, the pushchair also maintained its horizontal position-making a slight turn around its vertical axis, but without moving 
significantly towards the aisle. The maximum values for the dummy's head and chest were well within the limits defined by the child injury criteria. However, neck extension exceeded the allowed limit (10.51 Nm versus a tolerance limit of $8.31 \mathrm{Nm}$ ). This extension peak occurred when the pushchair sidebars struck the panel (at $188 \mathrm{~ms}$ ), exceeding the allowable limit for damage to the neck by $6.44 \%$. This vertical movement caused the highest values of $N_{i j}$ (traction-extension) and $N_{i j}$ (compression-extension) with values of $59.94 \%$ and $56.30 \%$, respectively (well within the possible values). It was considered that the behaviour of the pushchair and the No. 3 prototype panel was correct in retaining the pushchair during impact;

- Test E06: This configuration considered as "correct use" had the aim to verify the behaviour of the widest pushchair when interacting with the wheelchair back-restraint system. An analysis of the images showed that the stability offered by the pushchair restraint was correct and it maintained verticality. During the rebound, the pushchair remained horizontal without moving towards the aisle.

The maximum values produced for the dummy's head and chest were within the limits defined by the child injury criteria. The largest value for the injury criteria appeared for the neck, corresponding to an extension in the $\mathrm{Y}$ direction, although the allowable limit values were not exceeded (3.87 Nm compared to a tolerance limit of $8.31 \mathrm{Nm}$ ). This peak occurred when the pushchair sidebars reached their greatest vertical deformation on striking the panel (at $138 \mathrm{~ms}$ ). However, only $44.15 \%$ of the allowable limit for avoiding damage to the neck was reached. It was considered that the behaviour of the pushchair and the wheelchair rear wall restraint system was correct as the pushchair was retained during impact.

From the analysis of the results obtained (Tables 2 and 3), it could be seen that in the tests in which a "misuse" configuration was used (E02, E03, E05), with a distance of $150 \mathrm{~mm}$ between the pushchair and the panel, and without brakes applied to the wheels, the most critical values corresponded to the extension moment in the neck in the $\mathrm{Y}$ direction - in which all the values were greater than $90 \%$ and two of the tests (E02 and E05) exceeded the injury tolerance limits.

In the trials that reproduced "correct use" conditions (E04, E06), where the pushchair was initially touching the panel and the brakes were applied to the wheels, the values of the moment extension in the $\mathrm{Y}$ direction were about $50 \%$ of the permitted value. This indicates the need to always place the pushchair in contact with the restraint in all cases. No potentially harmful values were obtained for the other tested parameters. Furthermore, the results of the dynamic tests have shown very consistent results in different areas of the body (i.e., if a test revealed a low-level acceleration in the head, then results also showed less stress on the neck and lesser accelerations on the chest).

A more detailed analysis of the results in terms of test configurations enabled other qualitatively interesting conclusions to be drawn. In fact, several comparative studies were performed in the project as we describe below.

\subsection{Analysis of E02 in Comparison with E03}

In this case, two test configurations, classified as misuse, were compared. In one test, the least stable pushchair (the SX) supported by the wheelchair back-restraint system (E02), was compared with the other test where the prototype No. 2 it was restrained (E03). Fig. 7 shows a superimposed image of the two configurations with the relative position of the pushchair during free flight of $150 \mathrm{~mm}$ towards each one of the restraint systems.

Although the distance of free flight in both cases is the same $(150 \mathrm{~mm})$, the difference in height between the two panels and the contact point of the pushchair with them, produces different behaviour in each case. Thus, while the contact of the pushchair with the wheelchair back-restraint occurs with the (horizontal) pushchair handgrip, contact with the ASUCAR folding panel occur lower down with the central telescopic rod 

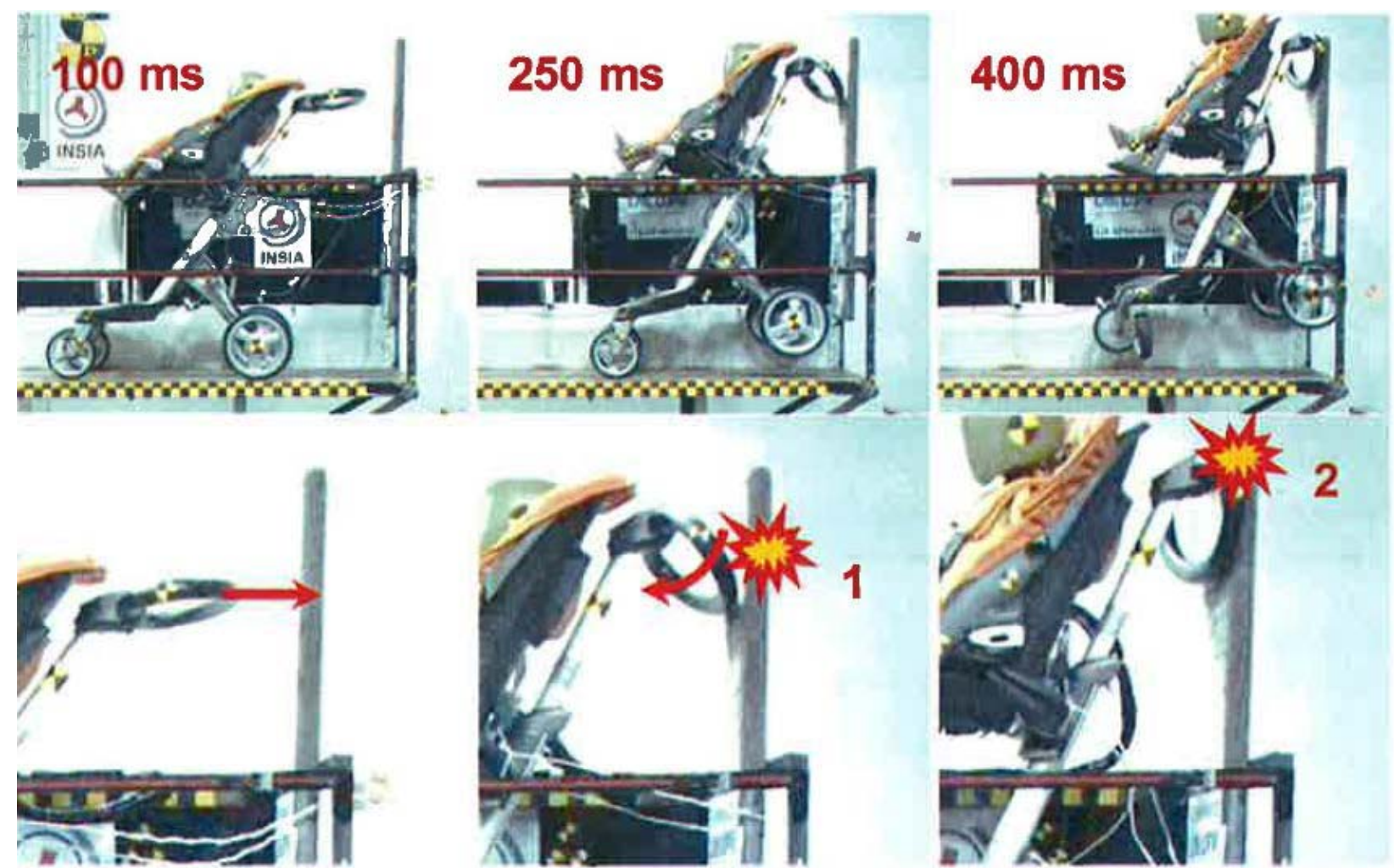

Fig. 6 Impact instant in test 13LSP0036E02.

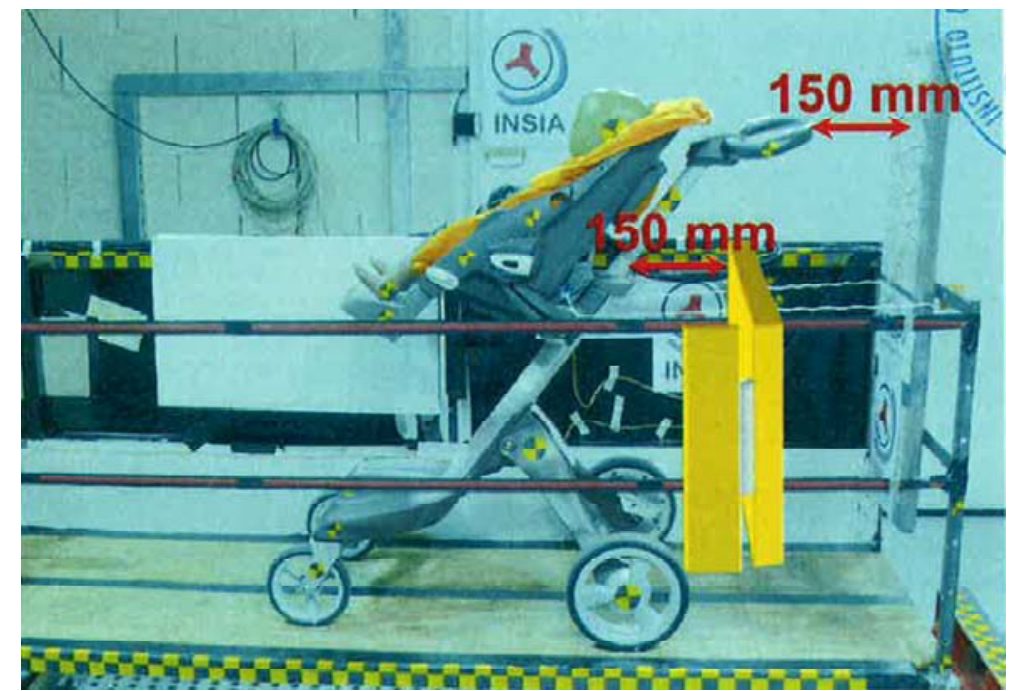

Fig. 7 Superimposed image of test configurations E02 (ASUCAR prototype No. 1) vs. E03 (fixed back-restraint panel for wheelchair passengers) using the SX pushchair.

on which the chair is mounted. The horizontal displacement of the pushchair in the case of the wheelchair back-restraint system is greater, due to the handgrip bends when it comes into contact with the panel and the pushchair continues travelling until the telescopic mast strikes the panel. As a consequence, all the values obtained in the test with the fixed wheelchair back-restraint system (E02) are higher than those obtained with the folding panel (E03).

The most critical parameter in both cases corresponds to the vertical extension of the dummy's neck (in direction Y). The limit was exceeded by $12 \%$ 
in the case of the fixed wheelchair back-restraint system (E02), while in the case of the folding ASUCAR panel (E03), values did not reach the limit of tolerance ( $5 \%$ below the limit).

\subsection{Analysis of E04 Compared with E05}

In this case, two configurations were tested using the same QB pushchair, one of the widest models with twin front wheels. The analysis compared a "correct" configuration (E04) in which the pushchair was in contact with the folding ASUCAR panel prototype No. 3 and wheel brakes applied, with a "misuse" application (E05) where the pushchair faced backwards, with a free flight distance of $150 \mathrm{~mm}$ to the folding panel, and wheel brakes did not apply.

The results show that all the parameters obtained for the E05 trial (misuse) were worse than those obtained for the E04 trial (correct use). Only a small gap of 150 $\mathrm{mm}$ between the pushchair and the panel caused all the registered levels to be double or more. The highest critical values exceeding the tolerance level were reached for the vertical extension of the neck, which exceeded the level of tolerated damage by $26 \%$. Values increased by up to four times for head and chest accelerations, although critical values were not exceeded.

\section{Conclusions}

This paper describes the dynamic tests made with pushchairs in various configurations. Three prototype pushchairs restraint devices and a fixed back-restraint system for wheelchair passengers were used. The tests were designed to verify the pushchair transportation configuration developed in the ASUCAR project [1]. As a consequence of the situations of "misuse" (brakes not applied and a free flight gap between the pushchair and panel), and "correct use" (brakes applied to wheels and pushchair touching the panel), the following conclusions were made:

The restraint system developed in the ASUCAR project is capable of withstand the dynamic low-g impact loads generated by a pushchair under the most unfavourable dimensional conditions (the tallest SX and the widest QB models), when considering the transport of a child of up to $15 \mathrm{~kg}$, and reproducing low severity collisions $(2 \mathrm{~g}, 20 \mathrm{~km} / \mathrm{h} \Delta \mathrm{V})$, representative of sudden braking event in urban buses.

In configurations of "misuse" (no brakes applied and a gap of at least $150 \mathrm{~mm}$ between the pushchair and panel), the dynamic parameter values increase by between two and five times in comparison with the configuration of correct use, and may exceed the critical values of damage tolerance, mainly in the neck vertical movement $-M_{y}$, extension moment values.

The safest configurations for transporting pushchairs are those in which the pushchair is in contact with the rear panel, the wheels brakes are applied, and the pushchair facing backwards. This "correct use" configuration minimise children body parts accelerations preventing them from injuries in case of low-g impact conditions.

It has been shown that the dynamic behaviour of the new pushchair restraint system developed is as effective as the back-restraint system already in use for wheelchair passenger's transportation on M2 and M3 type buses as establishes regulations $[5,6]$.

In case of articulated buses where there is more space in the passenger compartment, the new restraint system is compatible with the installation of a rigid back-restraint for wheelchair passengers - without sacrificing the space reserved for this system. So, this new pushchairs restraint system is a real alternative that complements and efficiently reconciles the use of public buses by different passengers with reduced mobility (wheelchair and pushchair users).

This research has been based on the development of dynamic tests of a low severity equivalent to the sudden hard braking manoeuvres commonly made by city buses. The results will be of interest to relevant regulatory bodies, particularly those related to the use of existing systems designed for wheelchair users and the bus safety industry. It is a task for future work to 
confirm effectiveness during more severe impacts $(\Delta \mathrm{V}$ $30-40 \mathrm{~km} / \mathrm{h}$, and decelerations of up to $6 \mathrm{~g}-8 \mathrm{~g}$ ). Another line of future research would involve analysing the behaviour of the new restraint with forward facing pushchairs, a wider range of pushchairs sizes and weights, and side-impact bus accidents.

\section{Acknowledgments}

This research was developed under the auspices of the Spanish Ministry of Science and Innovation - who funded the project ASUCAR (PET2008_0328) in the 2008-2011 National Research Programme. The development of the injury criteria used in this study was supported by the Spanish Ministry of Science and Innovation who funded the project "Children and Elderly Safety in Bus Accidents" (CESBA/SANCA) (TRA2011-26313) in the 2008-2011 National Research Programme.

\section{References}

[1] Dols, J., Aparicio, F., Alcalá, E., Pons, V., Martínez, L., Martín, A. L., and Valles, B. 2012. Code of Good Practice for Transporting Pushchairs on Buses. Valencia (Spain): Universitat Politècnica de València.

[2] Dols, J., Pons, V., Alcalá, E., Vallés, B., and Martín, A. 2013. "Analysis of Dynamic Behaviour and Safety of Baby Carriages in Public Transportation Buses." Transportation Research Part A: Policy and Practice 49: 1-9.

[3] Dols, J., Pons, V., Alcala, E., Martínez, L., Martín, A., and Valles, B. 2011. Sistema de Seguridad Pasivo para Carritos de Niños en Vehículos de Transporte Público
(Passive Safety System for Children's Pushchairs in Public Transportation Vehicles). University Polytechnic of Valencia and University Polytechnic of Madrid. Patent No. P-201131557. (in Spanish)

[4] EC. 2001. Directive 2001/85/EC of the European Parliament and of the Council of 20 November 2001 Relating to Special Provisions for Vehicles used for the Carriage of Passengers Comprising more than Eight Seats in Addition to the Driver's Seat, and Amending Directives 70/156/EEC and 97/27/EC.

[5] United Nations. 2009. Regulation No. 107. Addenda 106. Rev.6 to 1958 Agreement. Date into Force 10.06.14, "Uniform Provisions Concerning the Approval of Category M2 or M3 Vehicles with Regard to Their General Construction".

[6] ECE/TRANS/WP.29/GRSG/2013/9. 2012. Proposal for the 06 Series of Amendments to Regulation No. 107 (M2 and M3 Vehicles) to Provide a Specific Area in Buses of Class I, Allowing a Child to Remain Seated in His/Her Pram or Pushchair.

[7] SAE. 2007. Instrumentation for Impact Test-Part 1-Electronic Instrumentation. J211-1. Rev. Jul 2007. Society of Automotive Engineers.

[8] Economic Commission for Europe of the United Nations (UN/ECE). 2011. Regulation No 44 rev. 2. "Uniform Provisions Concerning the Approval of Restraining Devices for Child Occupants of Power-Driven Vehicles ('Child Restraint Systems')'.

[9] United Nations. 2007. Regulation No. 94 rev. 1, "Uniform Provisions Concerning the Approval of Vehicles with Regard to the Protection of the Occupants in the Event of a Frontal Collision".

[10] Mertz, H. J., Irwin, A. L., and Prasad, P. 2003. "Biomechanical and Scaling Bases for Frontal and Side Impact Injury Assessment Referent Values." Stapp Car Crash Journal 47: 155-88. 